\title{
A Theoretical Study of the Lifetime and Transport of Large Ionospheric Density Structures
}

\author{
R. W. SCHUNK AND J. J. SOJKA
}

Center for Atmospheric and Space Sciences, Utah State University, Logan

\begin{abstract}
Large-scale density structures are a common feature in the high-latitude ionosphere. They have been observed in the dayside cusp, polar cap, and nocturnal auroral region. Relative to background densities, the perturbations associated with large-scale structures vary from about $10 \%$ to a factor of 100 . The lifetime and transport characteristics of "large" ionospheric structures (factor of 10 to 100) were studied with the aid of a three-dimensional time-dependent ionospheric model. Both density depletions and enhancements were considered. A density structure was created at a specific location in the high latitude $F$ region and the subsequent evolution was followed for different seasonal and solar cycle conditions as well as for different orientations of the interplanetary magnetic field (IMF), i.e., different convection patterns. Depending on the IMF, horizontal plasma convection can cause an initial structure to break up into multiple structures of various sizes, remain as a single distorted structure, or become stretched into elongated segments. The lifetime of an $F$ region density structure depends on several factors, including the initial location where it was formed, the magnitude of the structure, season, solar cycle, and convection pattern (IMF). For example, in summer the effects of a large density structure can disappear in a few hours or last as long as 9 hours, while in winter the effects can persist for 24 hours. The passage of perturbed plasma flux tubes through sunlit and auroral regions can significantly increase the lifetime of plasma enhancements and can significantly reduce the lifetime of plasma depletions.
\end{abstract}

\section{INTRODUCTION}

During the last decade, significant progress has been made in understanding the formation and evolution of the major morphological features observed in the high-latitude ionosphere. This understanding has been reflected in the ability of the ionospheric models to successfully describe such features as the mid-latitude trough, the polar hole, the cross polar cap tongue of ionization, and the auroral ionization enhancements. The ionospheric models have also been successful in describing the variation of these features with solar cycle, season, and geomagnetic activity (convection pattern) and in describing the universal time (UT) dependence of these features that results from the displacement between the geomagnetic and geographic poles [cf. Knudsen et al., 1977; Watkins, 1978; Sojka et al., 1981a, b, c; 1982; Schunk and Sojka, 1982b; Sojka and Schunk, 1983, 1984, 1985; Schunk et al., 1986].

More recently, considerable attention has been directed towards studying the plasma density structures observed at high latitudes [Vickrey et al., 1980; Muldrew and Vickrey, 1982; Kelley et al., 1982; Buchau et al, 1983, 1985; Basu et al., 1983; Kelly and Vickrey, 1984; Weber and Buchau, 1985; Foster and Doupnik, 1984; Weber et al., 1984, 1985, 1986; de la Beaujardiere et al., 1985; Robinson et al., 1985]. There are small-scale $(\lesssim 1 \mathrm{~km})$, medium-scale $(\sim 10 \mathrm{~km})$ and large-scale $(\gtrsim 100 \mathrm{~km})$ density structures. These structures have been observed in the $E$ region, $F$ region, and topside ionosphere throughout the polar region, including the dayside cusp, polar cap, and auroral zone. The small-scale structures appear to be produced within and on the edges of the larger structures through plasma instabilities and are typically referred to as density irregularities [Fejer and Kelley, 1980; Kelley et al., 1982; Lee, 1984]. The medium and large-scale structures, which have also been called "blobs," "patches," and "enhancements," may have several source mechanisms. Some of the suggested mechanisms include structured particle precipitation in the dayside cusp, in sun-

\footnotetext{
Copyright 1987 by the American Geophysical Union.

Paper number 7 A9073

0148-0227/87/007A-9073\$05.00
}

aligned polar cap arcs, and in the nocturnal auroral oval [Kelley et al., 1982; Muldrew and Vickrey, 1982; Basu et al., 1983; Weber et al., 1984; Robinson et al., 1985]; structured electric fields which lead to structured enhanced loss rates [Kelly and Vickrey, 1984]; and the convection of plasma over large distances from both auroral source regions and the sunlit hemisphere [Kelly and Vickrey, 1984; Weber et al., 1984, 1986; Buchau et al., 1985; de la Beujardiere et al., 1985; Robinson et al., 1985].

The latter studies have clearly shown that plasma blobs can survive for a long time and can drift far from their source regions. As the blobs convect, their shape can change markedly, as shown by Robinson et al. [1985]. These authors considered a circular blob of plasma initially located at 1800 MLT and $75^{\circ}$ magnetic latitude and then followed the evolution of the blob as it moved in response to a Heelis et al. [1982] convection pattern. At later times, the plasma blob drifted to the equatorward edge of the auroral oval and became elongated along the boundary. The elongation was a result of the different convection times associated with various streamlines on which the blob was located. Although the authors did not solve for the temporal evolution of the blob density, the results, nevertheless, clearly showed the important role horizontal transport plays in the evolution of blob features.

Recently, we have used a time-dependent model of the highlatitude ionosphere in order to quantitatively study the origins, transport, and decay of large-scale $F$ region blobs [Sojka and Schunk, 1986]. The model study focused on blobs produced by particle precipitation in sun-aligned polar cap arcs, and it was found that the observed energy fluxes can readily account for blob formation if the plasma flux tubes are exposed to the precipitation for 5-10 min. The subsequent convection of the blob across the polar cap and into the nocturnal auroral oval produced a signature similar to that observed by incoherent scatter radars.

Although the above study was extremely useful in elucidating blob characteristics, it was limited to just one geophysical situation (winter and medium solar activity), one interplanetary magnetic field (IMF) configuration (i.e., one convection pat- 
tern), and the blobs were followed only until they reached the nightside subauroral region. In this study we focused attention on the lifetime and transport of large-scale high-density blobs for a range of solar cycle, seasonal, and IMF conditions. For given initial density enhancements, we followed the blobs until they disappeared and observed their spatial and temporal evolution.

\section{IONOSPHERE MODEL}

The ionospheric model was initially developed as a midlatitude, multi-ion $\left(\mathrm{NO}^{+}, \mathrm{O}_{2}^{+}, \mathrm{N}_{2}^{+}\right.$, and $\left.\mathrm{O}^{+}\right)$model by Schunk and Walker [1973]. The time-dependent ion continuity and momentum equations were solved as a function of altitude for a corotating plasma flux tube including diurnal variations and all relevant $E$ and $F$ region processes. This model was extended to include high-latitude effects due to convection electric fields and particle precipitation by Schunk et al. [1975, 1976]. A simplified ion energy equation was also added, which was based on the assumption that local heating and cooling processes dominate (valid below $500 \mathrm{~km}$ ). Flux tubes of plasma were followed as they moved in response to convection electric fields. A further extension of the model to include the minor ions $\mathrm{N}^{+}$and $\mathrm{He}^{+}$, an updated photochemical scheme, and the mass spectrometer/ incoherent scatter (MSIS) atmospheric model is described in Schunk and Raitt [1980].

The addition of plasma convection and particle precipitation models is described by Sojka et al. $[1981 a, b]$. More recently, the ionospheric model has been extended by Schunk and Sojka [1982a] to include ion thermal conduction and diffusionthermal heat flow, so that the ion temperature is now rigorously calculated at all altitudes between 120 and $1000 \mathrm{~km}$. The adopted ion energy equation and conductivities are those given by Conrad and Schunk [1979]. Also, the electron energy equation has been included recently by Schunk et al. [1986], and consequently, the electron temperature is now rigorously calculated at all altitudes. The electron energy equation and the heating and cooling rates were taken from Schunk and Nagy [1978], and the conductivities were taken from Schunk and Walker [1970].

With the high-latitude model, flux tubes of plasma are followed as they convect through a moving neutral atmosphere. Altitude profiles of the ion and electron temperatures and the $\mathrm{NO}^{+}, \mathrm{O}_{2}^{+}, \mathrm{N}_{2}^{+}, \mathrm{N}^{+}, \mathrm{O}^{+}$, and $\mathrm{He}^{+}$densities are obtained by solving the appropriate continuity, momentum, and energy equations including all of the high-latitude processes thought to be important. The equations are typically solved over the altitude range from 120 to $800 \mathrm{~km}$, with boundary conditions specified at the lower and upper ends of the flux tubes. For the densities, chemical equilibrium is assumed at $120 \mathrm{~km}$, and no escape flux is assumed at $800 \mathrm{~km}$. For the temperatures, local thermal coupling is assumed at $120 \mathrm{~km}$, and a specified heat flux is assumed at $800 \mathrm{~km}$. For this study the electron and ion heat fluxes through the upper boundary were taken to be zero.

\section{Lifetime and TRANSPORT OF Density Structures}

In this section we present ionospheric simulations dealing with the lifetime and transport of large-scale density structures for different solar cycle, seasonal, and IMF conditions. For given initial structures, we follow their evolution until they disappear in order to determine their lifetime and temporal/ spatial history. Specifically, the lifetime of a structure is defined as the time it takes for the structure density to decay to within
$10 \%$ of the background plasma density. Before we present results, however, it is necessary to describe the various inputs needed by our high-latitude ionospheric model.

There are several inputs that are needed for our ionospheric model, including the neutral atmosphere, thermospheric wind, plasma convection pattern, and an auroral oval. For the neutral atmosphere, we adopted the MSIS model [Hedin et al., 1977] with day 170 representing summer, day 329 representing winter, $F_{10.7}=190 \times 10^{-22} \mathrm{~W} \mathrm{~m}^{-2} \mathrm{~Hz}^{-1}$ for solar maximum, $F_{10.7}=70 \times$ $10^{-22} \mathrm{~W} \mathrm{~m}^{-2} \mathrm{~Hz}^{-1}$ for solar minimum, and $A p=10$ for all four cases. For the thermospheric wind, we adopted a simple pattern with an antisunward flow over the polar cap at a speed of about $200 \mathrm{~m} \mathrm{~s}^{-1}$. This wind pattern has been used in several of our previous studies and is described by Sojka et al. [1981a, b].

Electron precipitation in the auroral oval acts as a plasma production source, a source of bulk heating for the thermal electrons, and a source of heat that flows through our upper boundary. For our auroral oval, we adopted the empirical model developed by Spiro et al. [1982] and used an $A_{\epsilon}$ value of 350. Figure 1 (top) shows the auroral oval in an MLT-magnetic latitude reference frame. The adopted oval is approximately $10^{\circ}$ wide, with the strongest precipitation occurring in the night sector where the energy flux reaches $6.3 \mathrm{erg} \mathrm{cm}^{-2} \mathrm{~s}^{-1}$. The procedure for calculating the ionization rate and the thermal electron bulk heating rate from the auroral electron energy flux is described by Schunk et al. [1986].

\subsection{Dayside Structuring Source}

In the first series of simulations, we considered a dayside structuring source and adopted a two-cell convection pattern with antisunward flow over the polar cap. The pattern, which is shown in the top panel of Figure 1, corresponds to a symmetric, two-cell configuration of the Volland [1978] type with corotation added. This pattern is representative of moderate geomagnetic activity with $K p=3$ and a total cross polar cap potential of $62 \mathrm{kV}$. In the polar cap, the electric field is about $17 \mathrm{mV} \mathrm{m}^{-1}$ and the corresponding antisunward convection speed is about $300 \mathrm{~m}$ $\mathrm{s}^{-1}$. The solid line is our adopted "test" trajectory, which was used to follow convecting plasma flux tubes in different reference frames. In the middle panel, this test trajectory is followed for 3.5 traversals in a geographic inertial frame starting on the dayside between 1000 and 1100 LT (shown by the dot in Figure 1 (top)). The tick marks along the flux tube path show the elapsed time at hourly intervals. Three complete traversals of the test trajectory take about 27 hours. Although the 3.5 trajectory loops overlap in the magnetic frame, they do not overlap in the geographic inertial frame because the convection pattern is fixed in a magnetic frame and the magnetic pole rotates about the geographic pole. If a plasma blob were following this trajectory, it may or may not survive depending on how long it is exposed to ion production due to either auroral particle precipitation or solar EUV radiation (the terminators for summer and winter solstice are denoted by dashed lines). Therefore a satellite crossing the polar region may or may not see the plasma blob on successive passes. Finally, the bottom panel of Figure 1 shows the track of the plasma flux tube in a corotating geographic frame, which is the frame of reference in which ground-based measurements are made. In this frame, a blob of plasma drifting into view may appear to be coming from any direction, depending on the location of the observing site.

The test trajectory shown in Figure 1 was followed for 3.5 traversals starting on the dayside at the location marked by the 


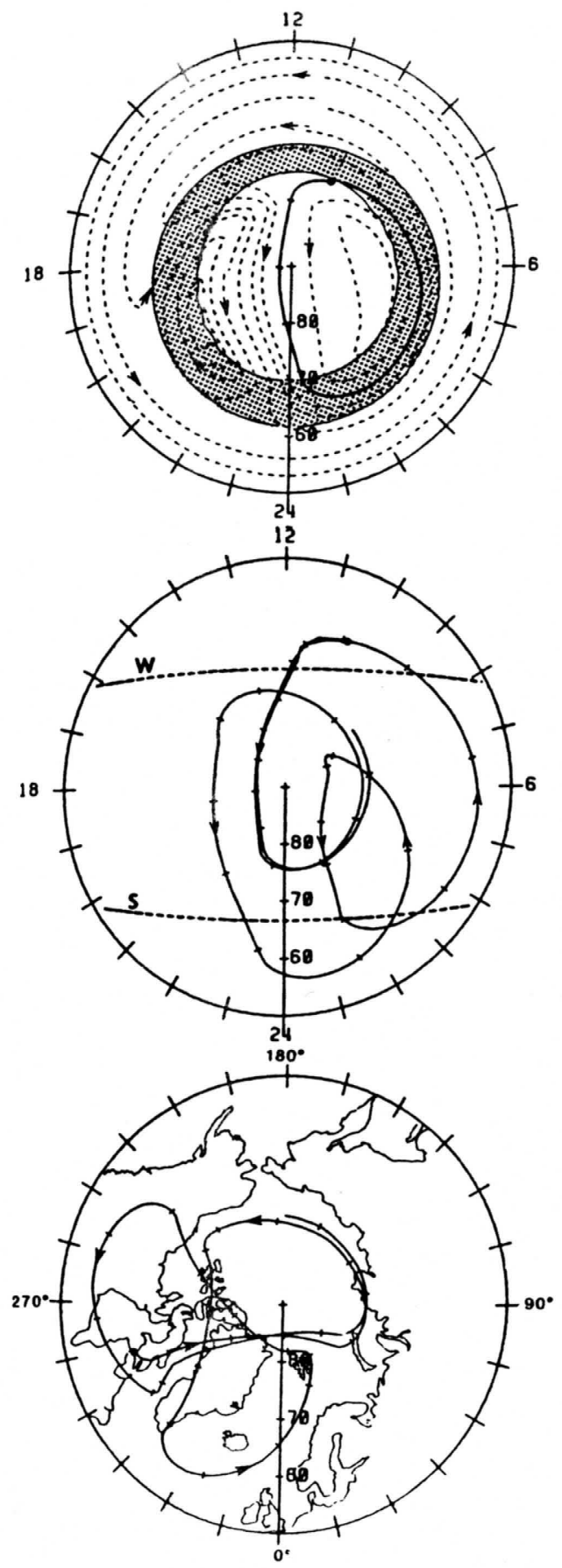

Fig. 1. Plasma drift trajectories in different coordinate systems for a Volland [1978] convection pattern with corotation added. The convection pattern is a symmetric two-cell pattern with a cross polar cap potential of $62 \mathrm{kV}$. The top panel shows the drift trajectories in a magnetic latitude-MLT reference frame. Also shown in this panel are a Spiroet al. [1982] auroral oval for $K p=3$ and a selected "test" trajectory (solid curve). The middle panel shows the path of a plasma flux tube in the geographic inertial frame for 3.5 traversals of the test trajectory. Also shown in the middle panel are the terminator locations for summer and winter solstice. The bottom panel shows the flux tube path in a path geographic reference frame. Tick marks along the flux tube path show the elapsed time at hourly intervals.

$\operatorname{dot}\left(285^{\circ} \mathrm{E}\right.$ longitude). Different seasonal and solar cycle cases Aere considered as well as different initial density perturbations. A start time of 1530 UT was selected so that the starting location was sunlit in both summer and winter. As a consequence, the background conditions at the starting location could be obtained from daytime steady state solutions. Figure 2 shows the variation of $N_{m} F_{2}$ along the test trajectory for three density distributions and for both winter and summer conditions at solar minimum. In both panels the solid curve corresponds to the natural plasma, while for the dotted and dashed curves the "initial" normal $\mathrm{O}^{+}$density profile was multiplied by factors of 10 and 100 , respectively. The variations of the solar zenith angle and the precipitating auroral electron energy flux along the test trajectory are shown at the top and bottom of each panel, respectively.

In winter the flux tube is in darkness most of the time, and therefore auroral precipitation is the only source of plasma along most of the test trajectory. For the natural case, $N_{m} F_{2}$ at the starting location is about $5 \times 10^{5} \mathrm{~cm}^{-3}$, but as the flux tube convects across the polar cap in darkness, $N_{m} F_{2}$ decreases until it reaches the nocturnal auroral oval. Because the test trajectory has a fairly long residence time in the oval, there are distinct $N_{m} F_{2}$ enhancements during each of the three traversals of the oval. When the initial $\mathrm{O}^{+}$density profile is multiplied by factors of 10 and 100 to create density structures (blobs), the subsequent plasma decay occurs at the same rate as the background plasma (solid curve) owing to the absence of sunlight. However, this is not true when the blob flux tubes enter the nocturnal auroral oval. Even though the blob densities are much greater than the background density when the flux tubes first encounter the nocturnal oval, auroral precipitation is sufficient to slow the decay of the blobs. Therefore in winter at solar minimum, the lifetime of a blob with a factor of 10 enhancement relative to the background plasma is about 11 hours, while for a factor of 100 enhancement the lifetime is about 19 hours.

In summer, the temporal evolution of the density structures is similar to that in winter, but there are some quantitative differences. First, for the natural case (solid curve), $N_{m} F_{2}$ at the dayside starting location is greater in winter than in summer

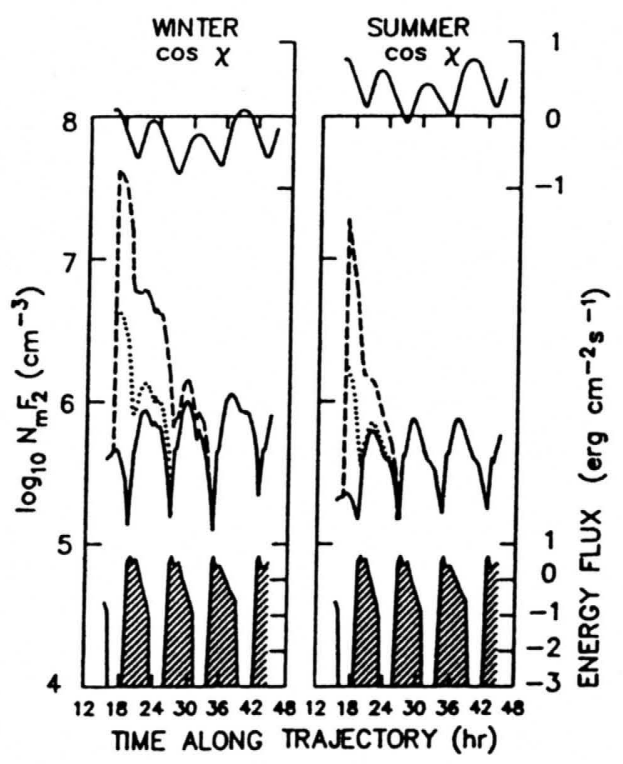

Fig. 2. $N_{m} F_{2}$ variation along the test trajectory for three plasma flux tubes in both summer and winter at solar minimum. The flux tubes start on the dayside at the location marked by the dot in Figure 1. The solid curve corresponds to the background plasma, while for the dotted and dashed curves the initial $\mathrm{O}^{+}$density profile was multiplied by factors of 10 and 100 , respectively. The solar 7 .nith angle variation along the trajectory is shown at the top, and the log of the auroral electron energy flux along the trajectory is shown at the bottom. 
owing to the well-known winter anomaly. The difference between summer and winter $N_{m} F_{2}$ 's is smaller in the auroral oval because we adopted the same precipitation pattern in summer and winter. With regard to the density structures, the important thing to note is that the lifetimes are much shorter in summer than in winter. The blob with a factor of 10 enhancement basically disappears in about 4 hours, while for the factor of 100 enhancement, the decay time is about 11 hours. The faster blob decay rate in summer is a result of both the change in the atmospheric $\mathrm{O} / \mathrm{N}_{2}$ density ratio and the greater importance of solar EUV production, which helps to maintain background plasma densities.

Figure 3 shows the $N_{m} F_{2}$ variation along the test trajectory for both winter and summer conditions at solar maximum. Except for the change from solar minimum to solar maximum, the cases shown in Figure 3 are the same as those in Figure 2. Comparing corresponding winter and summer cases, it is apparent that the seasonal trend is qualitatively the same at solar maximum and solar minimum. However, there are some quantitative differences. First, at solar maximum, the $N_{m} F_{2}$ values are larger outside of the oval and smaller inside the oval than those at solar minimum in both winter and summer. The net result is that auroral precipitation has a smaller perturbing effect on the background plasma at solar maximum than at solar minimum. Nevertheless, the blob lifetimes are similar at solar maximum to those obtained at solar minimum.

Figure 4 shows the temporal evolution of density depletions. In this figure the $N_{m} F_{2}$ variation along the test trajectory is plotted for three initial density distributions and for winter, solar maximum conditions. As in the previous cases, the flux tubes start on the dayside at the location marked by the dot in Figure 1. The solid curve corresponds to the natural situation and is the same as that shown in the left side of Figure 3, while the dotted and dashed curves correspond to cases for which the

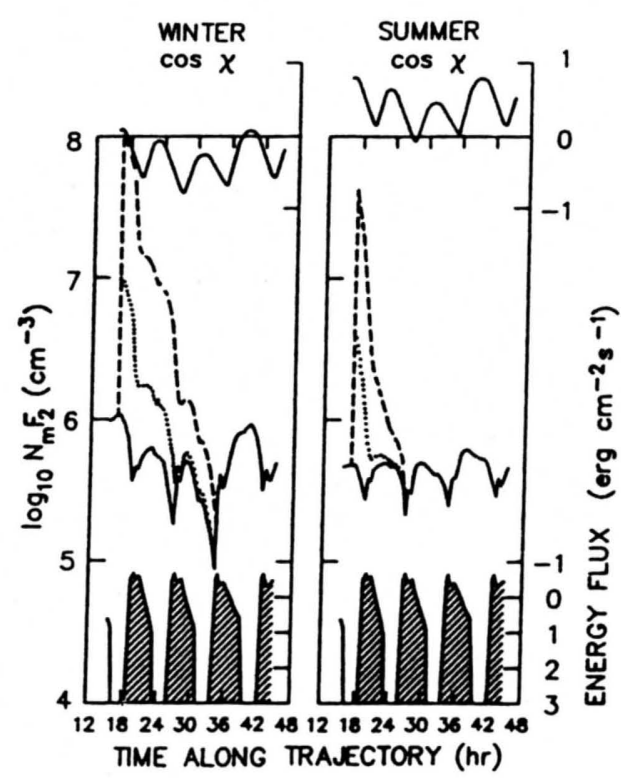

Fig. 3. $N_{m} F_{2}$ variation along the test trajectory for three plasma flux tubes in both summer and winter at solar maximum. The flux tubes start on the dayside at the location marked by the dot in Figure 1. The solid curve corresponds to the background plasma, while for the dotted and dashed curves the initial $\mathrm{O}^{+}$density profile was multiplied by factors of 10 and 100 , respectively. The solar zenith angle variation along the trajectory is shown at the top, and the log of the auroral electron energy flux along the trajectory is shown at the bottom.

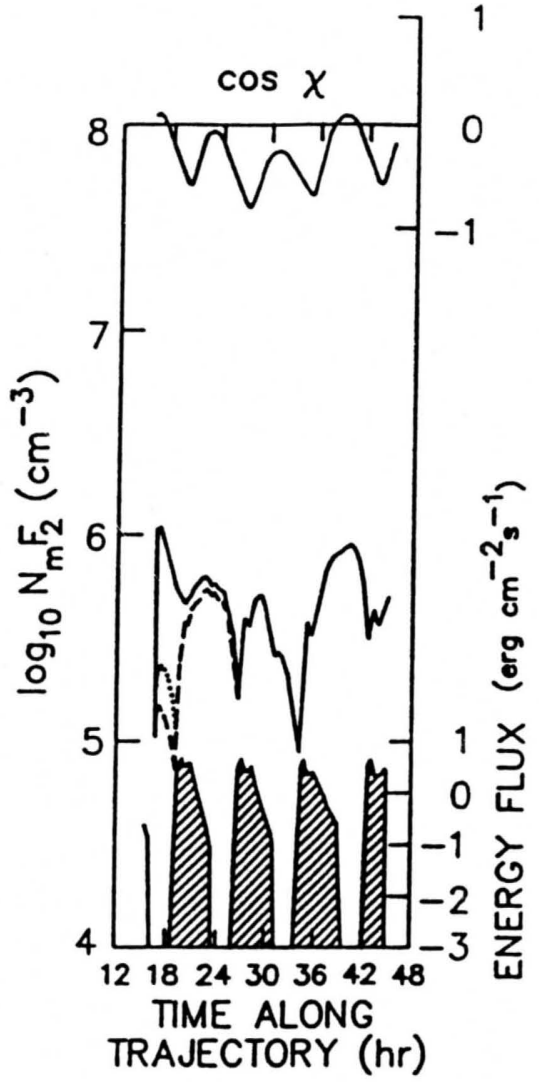

Fig. 4. $\quad N_{m} F_{2}$ variation along the test trajectory for three plasma flux tubes in winter at solar maximum. The flux tubes start on the dayside at the location marked by the dot in Figure 1. The solid curve corresponds to the background plasma, while for the dotted and dashed curves the initial $\mathrm{O}^{+}$density profile was multiplied by factors of 0.1 and 0.01 , respectively. Note that $N_{m} F_{2}$ does not track the initial $\mathrm{O}^{+}$density decrease because of the presence of molecular ions in the $F$ region. The solar zenith angle variation along the trajectory is shown at the top, and the log of the auroral electron energy flux along the trajectory is shown at the bottom.

initial $\mathrm{O}^{+}$density profile was reduced by successive factors of 10 . For both the natural and depleted flux tubes, the $N_{m} F_{2}$ values decrease as the plasma flux tubes convect across the polar cap in darkness. However, soon after entering the nocturnal auroral oval, the depleted flux tubes begin to fill and the $N_{m} F_{2}$ values quickly approach background values.

The lifetimes of depletions in summer are considerably shorter than in winter owing to the importance of ion production from solar EUV radiation. The depletions we considered typically had a lifetime of the order of a few hours. Also, the results we obtained at solar minimum were similar to those at solar maximum with regard to the lifetimes of depletions.

\subsection{Spatial Evolution of Structures}

In the previous subsection, we were primarily concerned with the lifetimes of structures with a range of enhanced densities. In this subsection, we consider a plasma blob with a given enhancement and a given spatial location, and then we follow the subsequent evolution of the blob as it drifts in response to convection electric fields for different IMF conditions.

The adopted blob was assumed to have a very large density enhancement of a factor of 100 relative to background $\mathrm{O}^{+}$densities and its initial location was taken to be in the dusk-midnight sector at $2000 \mathrm{MLT}$. Six plasma flux tubes along the $2000 \mathrm{MLT}$ 

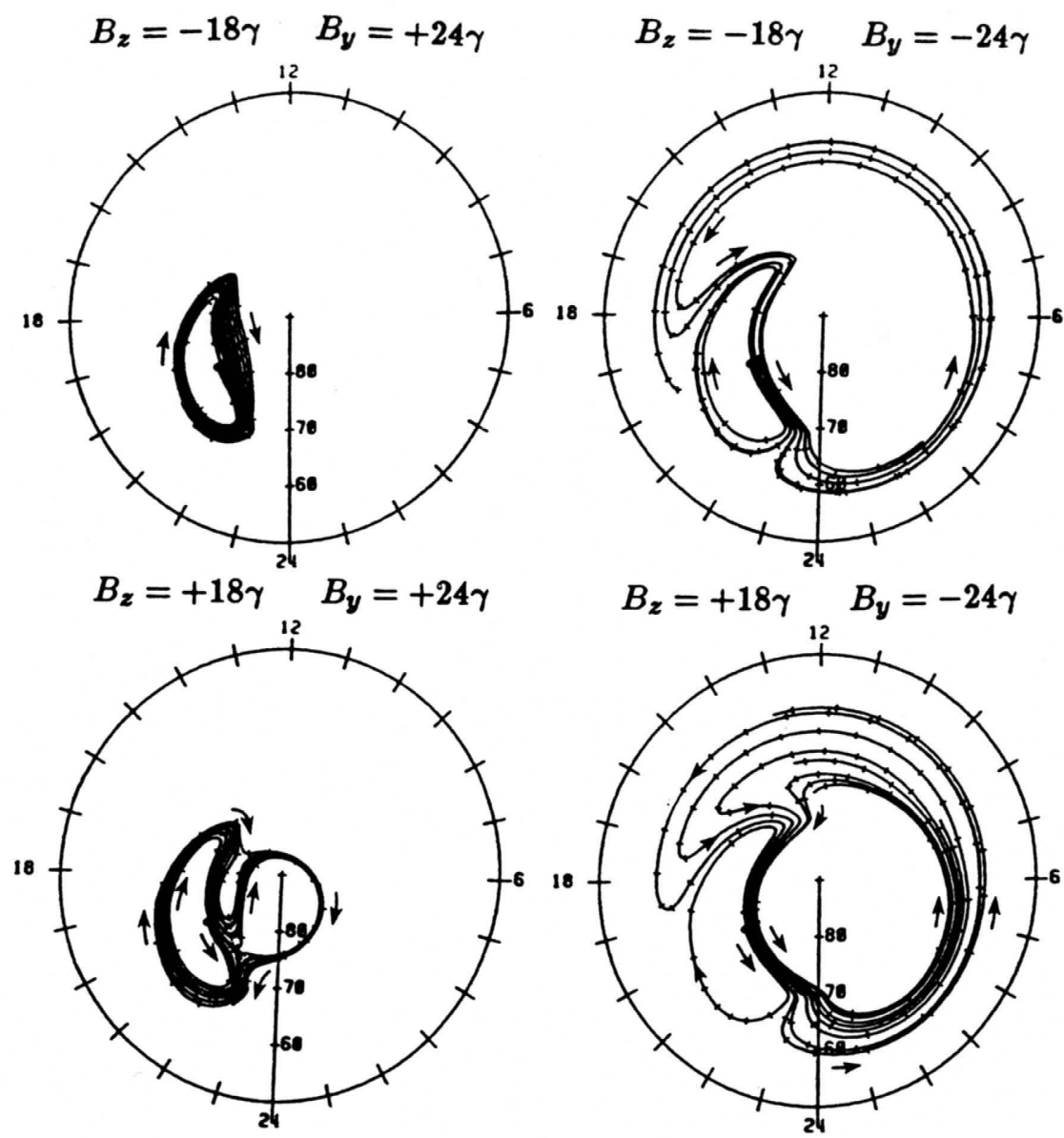

Fig. 5. Plasma convection trajectories in a magnetic latitude-MLT reference frame for different IMF conditions. The trajectories were obtained from the Sojka et al. [1986] convection patterns with corotation added. The top dials show $B_{y}$ positive and negative cases for southward IMF, while the bottom dials correspond to the same $\boldsymbol{B}_{y}$ cases for northward IMF. For each of the four IMF cases, six trajectories were followed for 24 hours starting at 2000 MLT and magnetic latitudes of $73.1^{\circ}, 74.3^{\circ}, 74.9^{\circ}, 75.5^{\circ}, 76.1^{\circ}$, and $76.7^{\circ}$. The tick marks along the trajectories represent hourly intervals.

meridian were selected to represent the initial width of the blob, with dipole latitude locations of $73.1^{\circ}, 74.3^{\circ}, 74.9^{\circ}, 75.5^{\circ}$, $76.1^{\circ}$, and $76.7^{\circ}$. These initial flux tube locations are shown in Figure 5 in a magnetic latitude-MLT reference frame. Also shown in Figure 5 is the subsequent spatial evolution of these plasma flux tubes for four different IMF configurations. The different cases were obtained from the Sojka et al. [1986] convection model with corotation added. The top figures show $B_{y}$ positive and negative cases for southward IMF, while the bottom figures correspond to the same $B_{y}$ cases for northward IMF. For southward IMF, two-cell convection patterns exist, while for northward IMF multicell convection exists. For $B_{y}$ positive, enhanced plasma convection occurs in the dawn sector, while for $B_{y}$ negative the enhanced plasma flow occurs in the dusk
sector.

It is apparent from Figure 5 that the IMF conditions have an important effect on the subsequent evolution of a density structure. For the case $\left(B_{z}=-18 \gamma, B_{y}=24 \gamma\right)$, the six trajectories stay together as they drift in response to convection electric fields. However, for the three other IMF configurations, the group of up into ses separate as they convect, i.e., the plasma blob breaks $-24 \gamma)$, one smaller blobs. For the case $\left(B_{z}=-18 \gamma, B_{y}=\right.$ tion cell, while anent of the initial blob stays in the dusk convecessentially co another segment crosses the polar cap and then essentially corotates throughout the morning and afternoon sectors before returning to the polar cap. For northward IMF, the breakup of the blob can be even more complicated because of the multicell nature of convection. For the case $\left(B_{z}=18 \gamma, B_{y}\right.$ $=24 \gamma$ ), one segment of the blob stays in the dusk convection cell, while another segment appears to circulate around the polar cap. For the last case $\left(B_{z}=18 \gamma, B_{y}=-24 \gamma\right)$, the bulk of the blob drifts around the midnight-dawn-noon sector, and as it does, it undergoes a significant spatial spreading and stretching (tick marks indicate hourly intervals).

A further comment is warranted with regard to the "breakup" of the blob. At $F$ region altitudes the plasma flow associated with the electrodynamic drift is approximately incompressible, and technically, a large blob cannot break apart even when streamlines separate. That is, in the idealized limit of incompressible flow, the blob segments will always be contiguous. However, small-scale structures imbedded in the global convection pattern and temporal variations of the electric field will act to break up the blob segments. Also, to a ground observer, the blob will "appear" to break up into segments. Therefore it is convenient to use terms such as breakup, segments, and stretching when describing blob dynamics.

Figure 6 shows the trajectories of the above plasma flux tubes in a corotating geographic reference frame. To an observer fixed to the ground, the situation could appear to be very complicated, depending on the IMF. Since different trajectories in the 

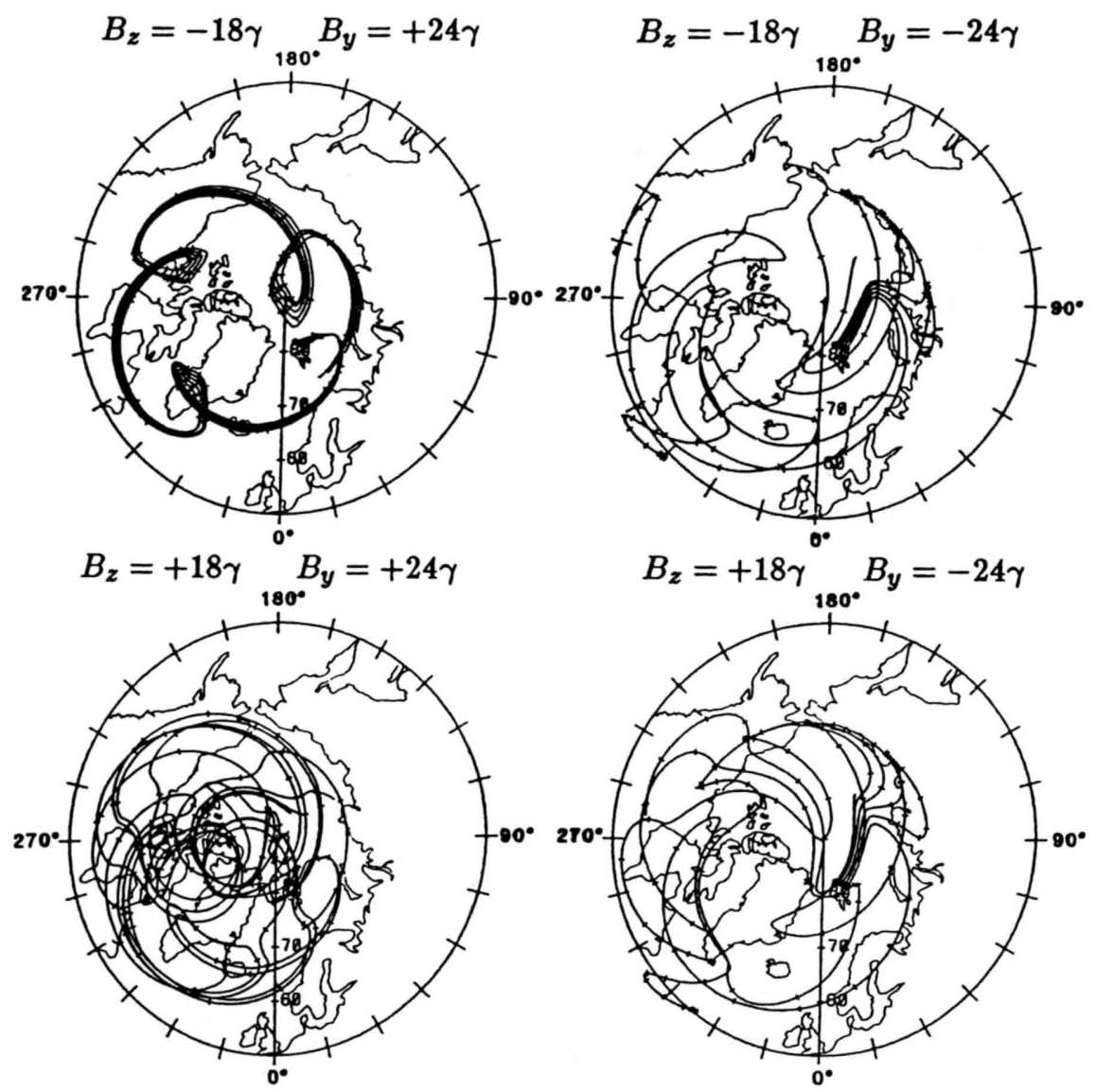

Fig. 6. Plasma convection trajectories in the corotating geographic frame for the different IMF cases shown in Figure 5.

initial blob cross each other at different, but later, times, a ground observer might think that the density enhancements observed at different times are unrelated. A further complication occurs because the later crossings of a ground site could be from different directions. It is apparent from Figure 6 that multiple observing sites and extended observations are needed to unambiguously determine blob dynamics, particularly for northward IMF.

With regard to observations, it should be noted that our modeling does not pertain to irregularities triggered by plasma instabilities. In this case, the situation is more complicated because the motion of a blob is usually the motion of the boundary of the irregularity as determined by the gradient in electron density. The motion of an irregularity boundary may be completely different than the motion of the plasma within the boundary.

Not only can an initial density structure break up into smaller segments, but the subsequent evolution of the individual segments can be very different, this is shown in Figure 7, where $N_{m} F_{2}$ is plotted versus time along five of the trajectories shown in Figure 5 for each of the four IMF cases and for winter and solar maximum conditions. The shading at the bottom of each plot indicates the times the convecting flux tubes are subjected to auroral precipitation. Dotted segments of the curves indicate the flux tubes are in darkness, while solid segments indicate sunlit conditions.

Considering first the $\left(B_{z}=-18 \gamma, B_{y}=24 \gamma\right)$ case (left column), it is readily apparent that the temporal evolution of $N_{m} F_{2}$ is very similar for the five trajectories because in this case the blob flux tubes stay together (see Figure 5). However, for the other southward IMF case $\left(B_{z}=-18 \gamma, B_{y}=-24 \gamma\right)$, only two of the five blob flux tubes stay sufficiently close to each other to have similar temporal histories (top two plots). For the other three flux tubes, the $N_{m} F_{2}$ variations are different because of different convection speeds along the trajectories, different exposures to auroral precipitation (see bottom of plots), and for one flux tube exposure to sunlight (solid curve segment). Note that the general conclusions reached above for southward IMF are also true for northward IMF (two columns on right). Finally, note that for a given IMF configuration and at a given time, the $N_{m} F_{2}$ values can be different by more than an order of magnitude even though the five blob flux tubes started out under the same conditions.

The extent to which seasonal changes affect blob histories is shown in Figure 8, where $N_{m} F_{2}$ variations along the five blob trajectories are plotted for summer and solar maximum conditions. Except for the change from winter to summer, the conditions leading to Figures 7 and 8 are identical. As was found for winter conditions, in summer the blob flux tubes have similar histories if they stay sufficiently close to each other (left column) and can have different histories if they separate (other IMF columns). However, for a given IMF, the $N_{m} F_{2}$ values along the five trajectories do not differ by a large amount in summer, while they did in winter (compare respective columns in Figures 7 and 8 ). This smaller $N_{m} F_{2}$ difference between trajectories in summer is a result of the greater importance of solar production, which acts to produce a more uniform background density distribution over the polar region. Consequently, auroral precipitation is not as important in summer as in winter, and after the initial rapid decay of the blob densities, the $N_{m} F_{2}$ histories 


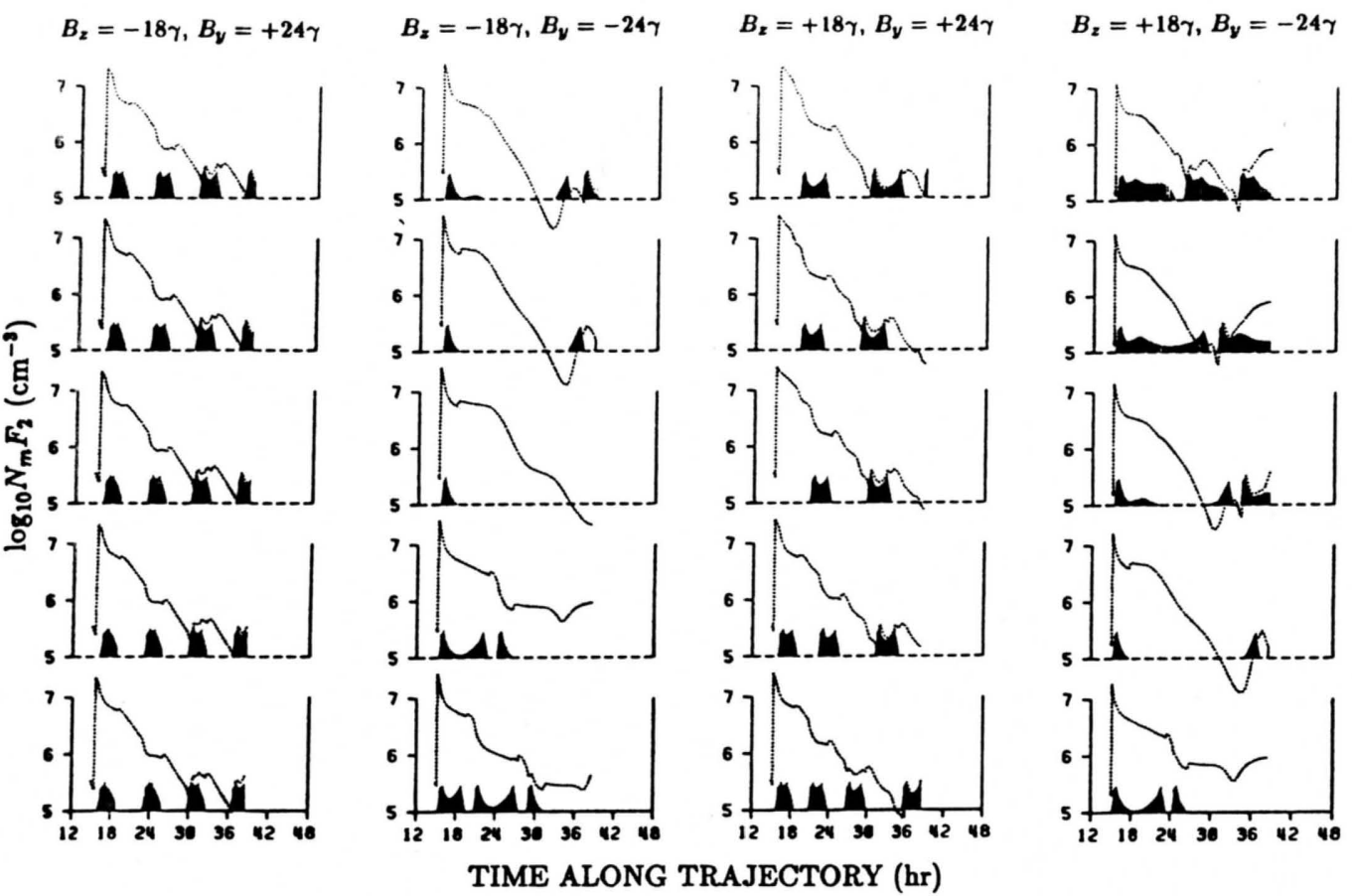

Fig. 7. $N_{m} F_{2}$ variation along five convection trajectories for four IMF cases in winter at solar maximum. The cases and trajectories are those shown in Figures 5 and 6. The shading at the bottom of each plot indicates the times the flux tubes are subjected to auroral precipitation. Dotted segments of the curves indicate the times the flux tubes are in darkness, while solid segments correspond to sunlit conditions.

are not that different in summer compared to what is found in winter.

\section{SUMMARY AND DiscusSion}

The lifetime and transport characteristics of large ionospheric structures were studied with the aid of a three-dimensional time-dependent ionospheric model. Both density enhancements and depletions were considered for a range of solar cycle, seasonal, and IMF conditions. For given initial density structures, we followed their evolution until they disappeared in order to determine their lifetime and temporal/spatial history. It was not possible to model every conceivable situation, and conse-
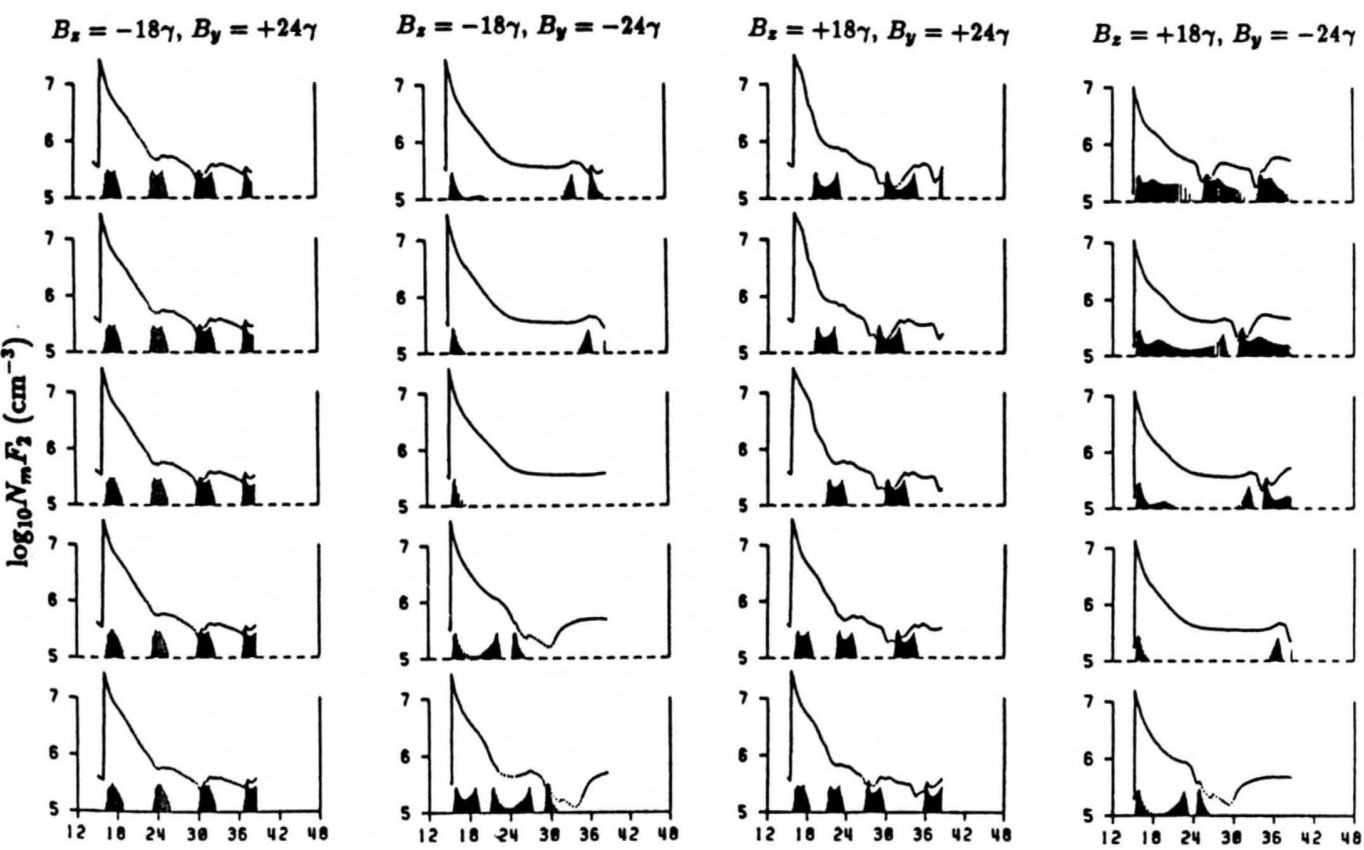

TIME ALONG TRAJECTORY (hr)

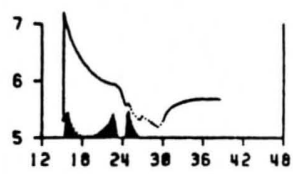

Fig. 8. $\quad N_{m} F_{2}$ variation along five convection trajectories for four IMF cases in summer at solar maximum. The cases and trajectories are those shown in Figures 5 and 6 . The shading at the bottom of each plot indicates the times the flux tubes are subjected to auroral precipitation. Dotted segments of the curves indicate the times the flux tubes are in darkness, while solid segments correspond to sunlit conditions. 
quently, we focused attention on large density perturbations (factors of 10 and 100) in order to determine maximum lifetimes. Also, only two blob locations were considered; a dayside structuring source and a dusk structuring source. Although many other locations could have been selected, the basic conclusions of our paper concerning the lifetime and transport characteristics of density structures would not change.

For the dayside structuring source, we adopted a two-cell convection pattern with antisunward flow over the polar cap. For a given initial perturbation, we followed the convecting flux tube until the perturbation disappeared. For the dusk structuring source, we considered a plasma blob with a given enhancement and a given spatial extent and we followed its subsequent spatial/temporal evolution as it drifted in response to convection electric fields for different IMF conditions. From these studies, we found the following.

1. In winter, the bulk of the polar region is in darkness most of the time, and therefore the blob and background densities tend to decay at the same rate, which acts to prolong the life of a blob. The lifetime of a blob with a factor of 10 enhancement relative to background densities is about 11 hours, while for a factor of 100 enhancement the lifetime is about 19 hours.

2. In summer, the lifetimes of density enhancements are much shorter than in winter. The blob with a factor of 10 enhancement basically disappears in about 4 hours, while for a factor of 100 enhancement the decay time is about 11 hours. The faster blob decay rate in summer is a result of both the change in the atmospheric $\mathrm{O} / \mathrm{N}_{2}$ density ratio and the greater importance of solar EUV production, which helps to maintain background plasma densities.

3. Auroral precipitation can further enhance a blob's density, depending on the residence time in the auroral oval.

4. The seasonal trend described above is qualitatively the same at solar maximum and solar minimum, and the blob lifetimes are similar. However, auroral precipitation has a smaller perturbing effect on the background plasma at solar maximum than at solar minimum.

5. In general, the lifetimes of density depletions are much shorter than density enhancements. A depleted flux tube tends to fill rapidly when it encounters ion production due to either auroral precipitation or solar EUV radiation. In summer, the depletions we considered typically had a lifetime of the order of a few hours.

6. Depending on the IMF, horizontal convection can cause an initial density structure to break up into multiple structures of various sizes, remain as a single distorted structure, or become stretched into elongated segments.

7. Not only can an initial density structure break up into smaller segments, but the subsequent evolution of the individual segments can be very different, depending on the IMF (convection pattern).

8. To an observer fixed to the ground, the identification of a blob source region may be difficult, particularly in winter when blob lifetimes are long. In this case, different trajectories in the initial blob can cross each other at different, but later, times and a ground observer might think that the density enhancements observed at different times are unrelated. Also, the later crossings of the ground site can be from different directions. However, part of the complexity is related to the rotation of the earth, which can be easily taken into account.

Numerous observations of plasma blobs, enhancements, and patches have been reported in recent years and various source mechanisms have been proposed. Kelley et al. [1982] suggested that the source region for plasma blobs is an annular ring along the poleward edge of the auroral oval, which represents the soff precipitation zone. Long-lived density enhancements would be produced in this region of soft particle precipitation, and sub. sequently, would convect equatorward and sunward, as observed by the radar measurements. However, Basu et al. [1983] pre. sented evidence for the creation of an $F$ region density enhance. ment near the equatorward edge of the auroral oval by direct soft particle precipitation during intense substorm conditions, Muldrew and Vickrey [1982] also showed evidence for a $15 \%$ increase in $N_{m} F_{2}$ in response to $\mathrm{keV}$ electron precipitation overa 12-min period. More recently, Robinson et al. [1985] proposed that the Harang discontinuity is an important source region for both large- and medium-scale ionization enhancements. They argued that slow convection in the Harang discontinuity would allow precipitation to act longer on the $F$ region plasma, which would lead to density enhancements. They also noted that the precipitation associated with the Harang discontinuity is tied to the convection pattern, and therefore the precipitation tracks the slow convection region even if the convection pattern changes. Consequently, in an average sense this favors the creation of ionization enhancements near midnight.

In addition to particle precipitation, there is mounting evidence that solar-produced plasma in combination with antisunward convection can act to create ionization patches in the polar cap and nocturnal auroral zone. Using a comprehensive data set composed of incoherent scatter radar data from European Incoherent Scatter (EISCAT), Chatanika, and Millstone Hill, and DE auroral images and convection velocities, de la Beaujardiere et al. [1985] presented convincing evidence that the $F$ region density enhancements they observed in the midnight sector were produced by solar EUV radiation in the day sector and transported across the polar cap. They also showed that the observed differences in the $F$ region densities at the three radars were related to longitudinal effects associated with the displacement between the geographic and geomagnetic poles. Buchau et al. [1985] also presented evidence that patches of ionization originate equatorward of the dayside cusp in the solar-produced ionosphere and are then convected into the polar cap. All-sky imaging and ionospheric sounding in the polar cap near the dayside cusp have clearly shown the emergence of patches from the cusp region [Weber and Buchau, 1985; Weber et al., 1984, 1986]. Also, incoherent scatter radar measurements from both Chatanika [Foster and Doupnik, 1984] and Sondrestrom [Kelly and Vickrey, 1984] have shown patches of ionization convecting poleward through the cusp and into the polar cap, which supports the concept of a solarproduced source for plasma blobs and patches.

The above experimental results indicate that both solarproduced and precipitation sources can lead to the creation of both large- and medium-scale ionization enhancements. It is also clear that the density enhancements can be transported over great distances, and consequently, the subsequent spatial/ temporal evolution of the density enhancement depends on the plasma convection pattern. Indeed, in one example, Robinson et al. [1985] showed how a density blob created at a specific location in the polar cap could be convected to the equatorward edge of the auroral oval and become elongated along the boundary; these results were obtained with a Heelis et al. [1982] model. However, our results show that the subsequent evolution of a plasma blob is very sensitive to the assumed convection pattern and that widely different results can be obtained by assuming different convection patterns or different locations for the blob source region. This is further complicated by the fact that the IMF can change markedly during a typical blob life- 
time. Nevertheless, our results support and enhance the conclusion reached by Robinson et al. [1985] that convection can lead to additional structuring of an initial plasma blob. We also showed that the lifetime and transport characteristics of a blob vary with season and solar cycle. In winter, blob lifetimes can be very long and multiple crossings of a ground site are possible, with later crossings coming from different directions. Consequently, multiple observing sites in the polar region and extended observations are needed to unambiguously determine the dynamics and lifetimes of ionization blobs, patches, and enhancements. We hope to have access to the required data in the near future so that we can conduct a detailed quantitative study of such features.

Acknowledgments. This research was supported by AFOSR contract F49620-86-C-0109, NASA grant NAGW-77, and NSF grant ATM-8417880 to Utah State University. The computer modeling effort was supported by the National Center for Atmospheric Research, which is sponsored by the NSF.

The Editor thanks R. M. Robinson and E. J. Weber for their assistance in evaluating this paper.

\section{REFERENCES}

Basu, S., E. MacKenzie, S. Basu, H. C. Carlson, D. A. Hardy, F. J. Rich, and R. C. Livingston, Coordinated measurements of low energy electron precipitation and scintillations/TEC in the auroral oval, Radio Sci., 18, 1151, 1983.

Buchau, J., B. W. Reinisch, E. J. Weber, and J. G. Moore, Structure and dynamics of the winter polar cap $F$ region, Radio Sci., 18, 995, 1983.

Buchau, J., E J. Weber, D. N. Anderson, H. C. Carlson, Jr., J. G. Moore, B. W. Reinisch, and R. C. Livingston, Ionospheric structures in the polar cap: Their origin and relation to $250-\mathrm{MHz}$ scintillation, Radio Sci., 20, 325, 1985.

Conrad, J. R., and R. W. Schunk, Diffusion and heat flow equations with allowance for large temperature differences between interacting species, J. Geophys. Res., 84, 811, 1979.

De la Beaujardiere, O., J. D. Craven, V. B. Wickwar, G. Candal, J. M. Holt, L. A. Frank, L. H. Brace, D. S. Evans, and J. D. Winningham, Universal time dependence of nighttime $F$ region densities at high latitudes, J. Geophys. Res., 90, 4319, 1985.

Fejer, B. G. and M. C. Kelley, Ionospheric irregularities, Rev. Geophys., 18, 401, 1980.

Foster, J. C., and J. R. Doupnik, Plasma convection in the vicinity of the dayside cleft, J. Geophys. Res., 89, 9107, 1984.

Hedin, A. E., C. A. Reber, G. P. Newton, N. W. Spencer, H. C. Brinton, H. G. Mayr, and W. E. Potter, A global thermospheric model based on mass spectrometer and incoherent scatter data, MSIS 2, Composition, J. Geophys. Res., 82, 2148, 1977.

Heelis, R. A., J. K. Lowell, and R. W. Spiro, A model of the highlatitude ionospheric convection pattern, J. Geophys. Res., 87, 6339, 1982.

Kelly, J. D., and J. F. Vickrey, $F$ region ionospheric structure associated with antisunward flow near the dayside polar cusp, Geophys. Res. Lett., 11, 907, 1984.

Kelley, M. C., J. F. Vickrey, C. W. Carlson, and R. Torbert, On the origin and spatial extent of high-latitude $F$ region irregularities, $J$. Geophys. Res., 87, 4469, 1982.

Knudsen, W. C., P M. Banks, J. D. Winningham, and D. M. Klumpar, Numerical model of the convecting $F_{2}$ ionosphere at high latitudes, $J$. Geophys. Res., 82, 4784, 1977.

Lee, M. C., Ohmic dissipation of Pederson current as the cause of high-latitude $F$ region ionospheric irregularities, J. Geophys. Res., $89,2289,1984$.

Muldrew, D. B., and J. F. Vickrey, High latitude $F$ region irregularities observed simultaneously with ISIS- 1 and the Chatanika radar, $J$. Geophys. Res., 87, 907, 1982.

Robinson, R. M., R. T. Tsunoda, J. F. Vickrey, and L. Guerin, Sources of $F$ region ionization enhancements in the nighttime auroral zone, $J$. Geophys. Res., 90, 7533, 1985.

chunk, R. W., and A. F. Nagy, Electron temperatures in the $F$ region of the ionosphere: Theory and observations, Rev. Geophys., 16, 355, 1978.

Schunk, R. W., and W. J. Raitt, Atomic nitrogen and oxygen ions in the daytime high-latitude $F$ region, J. Geophys. Res., 85, 1255, 1980.
Schunk, R. W., and J. J. Sojka, Ion temperature variations in the daytime high-latitude $F$ region, J. Geophys. Res., 87, 5169, $1982 a$.

Schunk, R. W., and J. J. Sojka, Ionospheric hot spot at high latitudes, Geophys. Res. Lett., 9, 1045, $1982 b$.

Schunk, R. W., and J. C. G. Walker, Transport properties of the ionospheric electron gas, Planet. Space Sci., 18, 1535, 1970.

Schunk, R. W., and J. C. G. Walker, Theoretical ion densities in the lower ionosphere, Planet. Space Sci., 21, 1875, 1973.

Schunk, R. W., W. J. Raitt, and P. M. Banks, Effect of electric fields on the daytime high-latitude $E$ and $F$ regions, J. Geophys. Res., 80, 3121,1975

Schunk, R. W., P. M. Banks, and W. J. Raitt, Effects of electric fields and other processes upon the nighttime high latitude $F$ layer, $J$. Geophys. Res., 81, 3271, 1976.

Schunk, R. W., J. J. Sojka, and M. D. Bowline, Theoretical study of the electron temperature in the high-latitude ionosphere for solar maximum and winter conditions, J. Geophys. Res., 91, 12,041, 1986.

Sojka, J. J., and R. W. Schunk, A theoretical study of the high-latitude $F$ region's response to magnetospheric storm inputs, J. Geophys. Res., 88, 2112, 1983.

Sojka, J. J., and R. W. Schunk, A theoretical $F$ region study of ion compositional and temperature variations in response to magnetospheric storm inputs, J. Geophys. Res., 89, 2348, 1984.

Sojka, J. J., and R. W. Schunk, A theoretical study of the global $F$ region for June solstice, solar maximum, and low magnetic activity, J. Geophys. Res., 90, 5285, 1985.

Sojka, J. J., and R. W. Schunk, A theoretical study of the production and decay of localized electron density enhancements in the polar ionosphere, J. Geophys. Res., 91, 3245, 1986.

Sojka, J. J., W. J. Raitt, and R. W. Schunk, A theoretical study of the high-latitude winter $F$ region at solar minimum for low magnetic activity, J. Geophys. Res., 86, 609, $1981 a$.

Sojka, J. J., W. J. Raitt, and R. W. Schunk, Theoretical predictions for ion composition in the high-latitude winter $F$ region for solar minimum and low magnetic activity, J. Geophys. Res., 86, 2206, $1981 b$.

Sojka, J. J., W. J. Raitt, and R. W. Schunk, Plasma density features associated with strong convection in the winter high-latitude $F$ region, J.Geophys. Res., 86, 6908, 1981 c.

Sojka, J. J., R. W. Schunk, and W. J. Raitt, Seasonal variations of the high-latitude $F$ region for strong convection, J. Geophys. Res., 87, 187,1982

Sojka, J. J., C. E. Rasmussen, and R. W. Schunk, An IMF dependent model of the ionospheric convection electric field, J. Geophys. Res., 91, 11281, 1986.

Spiro, R. W., P. H. Reiff, and L. J. Maher, Precipitating electron energy flux and auroral zone conductances: An empirical model, J. Geophys. Res., 87, 8215, 1982.

Vickrey, J. F., C. L. Rino, and T. A. Potemra, Chatanika/TRIAD observations of unstable ionization enhancements in the auroral F region, Geophys. Res. Lett., 7, 789, 1980.

Volland, H., A model of the magnetospheric electric convection field, $J$. Geophys. Res., 83, 2695, 1978.

Watkins, B. J., A numerical computer investigation of the polar $F$ region ionosphere, Planet. Space Sci., 26, 599, 1978.

Weber, E. J., and J. Buchau, Observation of plasma structure and transport at high latitudes, in The Polar Cusp, edited by J. A. Holtet and A. Egeland, p. 279, D. Reidel, Hingham, Mass., 1985.

Weber, E. J., J. Buchau, J. G. Moore, J. R. Sharber, R. C. Livingston, J. D. Winningham, and B. W. Reinisch, $F$ layer ionization patches in the polar cap, J. Geophys. Res, 89, 1683, 1984.

Weber, E. J., R. T. Tsunoda, J. Buchau, R. E. Sheehan, D. J. Strickland, W. Whiting, and J. G. Moore, Coordinated measurements of auroral zone enhancements, J. Geophys. Res., 90, 6497, 1985.

Weber, E. J., J. A. Klobucher, J. Buchau, H. C. Carlson, R. C. Livingston, O. de la Beaujardiere, M. McCready, J. E. Moore, and G. J. Bishop, Polar cap $F$ layer patches: Structure and dynamics, J. Geophys. Res., 91, 12,121, 1986.

R. W. Schunk and J. J. Sojka, Center for Atmospheric and Space Sciences, Utah State University, Logan, UT 84322. 